\title{
Public Health Concerns of Taenidae and Their Metacestodes
}

\section{Oryan $\mathrm{A}^{*}$ and Alidadi S}

Department of Pathology, School of Veterinary Medicine, Shiraz University, Shiraz, Iran

*Corresponding author: Oryan A, Department of Pathology, School of Veterinary Medicine, Shiraz University, Shiraz, Iran; Phone: +45 38623032 ; Fax: +45 3862 6974 ; E-mail: oryan@shirazu.ac.ir

Received date: November 01, 2014, Accepted date: November 07, 2014, Published date: November 13, 2014

Copyright: (C) 2014 Oryan A et al. This is an open-access article distributed under the terms of the Creative Commons Attribution License, which permits unrestricted use, distribution, and reproduction in any medium, provided the original author and source are credited.

\section{Editorial}

Tapeworms of the family Taeniidae are transmitted from the definitive hosts such as carnivores to the intermediated hosts including herbivores or omnivores and human beings via oral-fecal cycle $[1,2]$. This family includes two major genera namely Taenia and Echinococcus. The Taenia species include T. saginata, T. solium, T. asiatica, T. hydatigena, T. ovis, T. multiceps, T. serialis, T. pisiformis, T. taeniaeformis, and $T$. crassiceps $[2,3]$. In many endemic areas the diseases caused by the genus Taenia in humans are often categorised as neglected tropical diseases [3]. In general, the larval stages or metacestodes belonging to these tapeworms include hydatid cyts, cysticerci and coenuri [2]. All these species except for $T$. hydatigena (C. tenuicollis), T. ovis (Cysticercus ovis) and T. pisiformis ( $C$. pisiformis), cause zoonotic parasitic diseases and thereby are of public health importance [2-5]. Humans acquire infection by inadvertent consumption of ova or larval stages (metacestode) present in undercooked meat [3]. Cysticerus bovis, the metacestode of $T$. saginata, occurs only in beef and humans are only the definitive hosts and receive the infection by ingestion of the raw meat containing the cysticeri $[1,6,7]$. Neurocysticercosis is regarded as the most common zoonotic parasitic disease of the central nervous system (CNS) caused by $T$. solium metacestodes called Cysticercus cellulosae affecting human beings as the definitive and also aberrant hosts $[7,8]$. Indeed, human acquire this metacestode via ingestion of undercooked pork infected with cysticerci. On the other hand, the eggs hatched in the human intestine can finally produce the cysts in areas with high blood flow such as CNS (neurocysticercosis), the muscles (human cysticercosis), the subcutaneous tissue and the eye (subcutaneous and ocular cysticercosis, respectively) $[5,8]$. Taenia asiatica, also known as Asian Taenia, similar to T. saginata is responsible for intestinal teniasis and has been identified in several Asian countries [3,9]. Cysticercus fasciolaris is the larval stage of the cestode $T$. taeniaeformis in wide variety of small rodents, and occasionally birds and humans, as intermediate hosts with cats as the definitive hosts [10]. Adult forms of T. taeniaeformis and $C$. fasciolaris have been recognized from the intestine and liver of humans, respectively. Nonetheless, it seems that these cestodes are associated with low health risk to humans [10]. Coenurosis occurs in cerebral and non-cerebral forms. The former is caused by the larval stage of $T$. multiceps which possesses a metacestode stage namely Coenurus cerebralis $[2,11,12]$. T. gaigeri with its metacestode termed $C$. gaigeri causes non-cerebral coenurosis with cysts in the muscles of the intermediate hosts including herbivores as well humans [12-15]. It is unclear and debatable that $C$. cerebralis and $C$. gaigeri are the same or different species and the researches are focusing and performing detailed studies to answer this question $[11,12]$. Coenurus serialis, the larval stage of the cestode $T$. serialis are found as fluid-filled cystic masses in the muscles and subcutis of rodents and rarely humans as the intermediate hosts. This cestode, in many characteristics, is similar to T. multiceps [16]. T. crassiceps is intestinal tapeworm of carnivores forming the cyst-like larvae or metacestodes (cysticerci) in the body cavities and subcutaneous tissues of rodents as the intermediate hosts $[3,17]$. The muscles and subcutis of the immunosuppressed humans and the eye and cerebellum in immunocompetent ones are involved by the cestode larvae as tumor-like masses [17]. Four Echinococcus species are known to infect the human hosts during their larval stage including $E$. granulosus, E. multilocularis, E. oligarthus, and E. vogeli that the latter two species are associated with neotropical echinococcosis [18].

Hydatid disease or cystic echinococcosis is caused by the larval stage of E. granulosus associated with the existence of the cysts in visceral organs especially the liver and the lungs $[2,19]$. This cestode with its metacestode is recognized as the most important helminthic zoonoses and is of great public health and economic importance especially in tropical and developing countries [19,20]. Considering to its importance, treatment modalities for patients with the disease include chemotherapy via albendazole and mebendazole or praziquantel, puncture aspiration injection reaspiration (PAIR) and surgery [20,21]. Alveolar echinococcosis, a parasitic disease with public health importance, is caused by the cestode E. multilocularis transmitted between intermediate hosts such as rodents and definitive host including wild carnivores especially foxes. In humans, the metacestodes proliferate and form tumor-like masses causing organ dysfunction $[22,23]$. The disease if remains untreated, can lead to the death of the patient due to unlimited proliferation and metastasis of the lesions [23]. Polycystic echinococcosis caused by the metacestodes of $E$. vogeli and E. oligarthus is regarded as an emerging parasitic zoonotic disease with public health concern in the humid tropical rainforests [24]. The intermediate hosts for E. oligarthus include wild rodents with the development of cysts in the muscles, subcutaneous tissues or lungs, liver and spleen. In human cases, they have been found in the heart muscle, behind the eyes and the liver [18]. Diagnostic methods mostly include serologic tests such as ELISA, imaging and scanning techniques like magnetic resonance imaging (MRI), computed tomography (CT) and ultrasound [21]. Given high public health significance of these tapeworms, it needs to control. An effective preventive program and also early diagnosis should be designed and implemented and an appropriate treatment is necessary. Development of an effective, safe and cheap vaccine against the parasites can be helpful $[5,21]$. On the other hand, close proximity of the final hosts such as dogs to humans in these cases may be a main factor in the occurrence of human cases of the disease [23,25]. Therefore, dogs should be considered as the main source of infection and the major risk factor. The big problem for control of these diseases is reducing the risk factors including access of the stray dogs and other wild carnivores to the infected carcass wastes, consumption of raw meat and unwashed vegetables, poor sanitation, use of human feces as fertilizer, and inadequate meat inspection $[2,5]$. Regular deworming of carnivorous pets and repeated treatment of dogs (the major final host 
in most cases) with anti-parasitic agents, public awareness of different ways of parasite transmission, accuracy in carcass inspection, health education for dog owners, proper condemnation of the infected carcass to reduce the stray dog population, all can be useful in reducing the prevalence and incidence of these zoonotic parasitic diseases $[2,4,5,25]$.

\section{References}

1. Oryan A, Moghaddar N, Gaur SN (1995) Taenia saginata cysticercosis in cattle with special reference to its prevalence, pathogenesis and economic implications in Fars Province of Iran. Vet Parasitol 57: 319-327.

2. Oryan A, Goorgipour S, Moazeni M, Shirian S (2012) Abattoir prevalence, organ distribution, public health and economic importance of major metacestodes in sheep, goats and cattle in Fars, southern Iran. Trop Biomed 29: 349-359.

3. Jia WZ, Yan HB, Guo AJ, Zhu XQ, Wang YC, et al. (2010) Complete mitochondrial genomes of Taenia multiceps, T. hydatigena and T. pisiformis: additional molecular markers for a tapeworm genus of human and animal health significance. BMC Genomics. 11: 447.

4. Oryan A, Moghaddar N, Gaur SN (1994) Metacestodes of sheep with special reference to their epidemiological status, pathogenesis and economic implications in Fars Province, Iran. Vet Parasitol 51: 231-240.

5. Van De N, Le TH2, Lien PT1, Eom KS2 (2014) Current status of taeniasis and cysticercosis in Vietnam. Korean J Parasitol 52: 125-129.

6. Oryan A, Gaur SN, Moghaddar N, Delavar H (1998) Clinico-pathological studies in cattle experimentally infected with Taenia saginata eggs. J S Afr Vet Assoc 69: 156-162.

7. Nunes Dda S, Gonzaga HT, Ribeiro Vda S, da Cunha JP Jr, Costa-Cruz JM (2013) Taenia saginata metacestode antigenic fractions obtained by ionexchange chromatography: potential source of immunodominant markers applicable in the immunodiagnosis of human neurocysticercosis. Diagn Microbiol Infect Dis. 762: 36-41.

8. Coral-Almeida M, Rodríguez-Hidalgo R2, Celi-Erazo M3, García HH4, Rodríguez S5, et al. (2014) Incidence of human Taenia solium larval Infections in an Ecuadorian endemic area: implications for disease burden assessment and control. PLoS Negl Trop Dis 8: e2887.

9. Parija SC, Ponnambath DK (2013) Laboratory diagnosis of Taenia asiatica in humans and animals. Trop Parasitol 3: 120-124.

10. Rodriguez-Vivas RI, Panti-May JA, Parada-Lopez J, Hernandez-Betancourt SF, Ruiz-Pina HA (2011) The occurrence of the larval cestode Cysticercus fasciolaris in rodent populations from the Cuxtal ecological reserve, Yucatan, Mexico. J Helminthol. 85: 458-461.

11. Oryan A, Nazifi S, Sharifiyazdi H, Ahmadnia S (2010) Pathological, molecular, and biochemical characterization of Coenurus gaigeri in Iranian native goats. J Parasitol 96: 961-967.
12. Oryan A, Akbari M, Moazeni M, Amrabadi OR (2014) Cerebral and noncerebral coenurosis in small ruminants. Trop Biomed 31: 1-16.

13. Moghaddar N, Oryan A, Gaur SN (1992) Coenurosis in cattle in Iran. J Appl Anim Res 2: 119-121.

14. Tafti AK, Oryan A, Maleki M (1997) Pathological changes due to coenurosis in a wild ewe in Iran. J Vet Parasitol 11: 65-68.

15. Kheirandish R, Sami M, Azizi S, Mirzaei M (2012) Prevalence, predilection sites and pathological findings of Taenia multiceps coenuri in slaughtered goats from south-east Iran. Onderstepoort J Vet Res 79: E1-5.

16. Schneider-Crease IA, Snyder-Mackler N, Jarvey JC, Bergman TJ (2013) Molecular identification of Taenia serialis coenurosis in a wild Ethiopian gelada (Theropithecus gelada). Vet Parasitol 198: 240-243.

17. Ntoukas V, Tappe D, Pfütze D, Simon M, Holzmann T (2013) Cerebellar cysticercosis caused by larval Taenia crassiceps tapeworm in immunocompetent woman, Germany. Emerg Infect Dis 19: 2008-2011.

18. Soares Mdo C, Rodrigues AL, Moreira Silva CA, Brito EM, Gomes-Gouvea MS, et al. (2013) Anatomo-clinical and molecular description of liver neotropical echinococcosis caused by Echinococcus oligarthrus in human host. Acta Trop. 125: 110-114.

19. Mehrabani D, Oryan A, Sadjjadi SM (1999) Prevalence of Echinococcus granulosus infection in stray dogs and herbivores in Shiraz, Iran. Vet Parasitol 86: 217-220.

20. Ahmadnia S, Moazeni M, Mohammadi-Samani S, Oryan A (2013) In vivo evaluation of the efficacy of albendazole sulfoxide and albendazole sulfoxide loaded solid lipid nanoparticles against hydatid cyst. Exp Parasitol 135: 314-319.

21. Oryan A, Alidadi S (2014) Cystic Echinococcosis: With emphasis on treatment options. Trop Med Surg 1: e112.

22. Kouguchi H, Matsumoto J, Nakao R, Yamano K, Oku Y, et al. (2013) Characterization of a surface glycoprotein from Echinococcus multilocularis and its mucosal vaccine potential in dogs. PLoS One 8: e69821.

23. Umhang G, Comte S, Raton V, Hormaz V, Boucher JM, et al. (2014) Echinococcus multilocularis infections in dogs from urban and peri-urban areas in France. Parasitol Res 113: 2219-2222.

24. Almeida F, Oliveira F1, Neves R2, Siqueira N3, Rodrigues-Silva R1, et al. (2014) Morphometric characteristics of the metacestode Echinococcus vogeli Rausch \& Bernstein, 1972 in human infections from the northern region of Brazil. J Helminthol .

25. Sharifiyazdi H, Oryan A, Ahmadnia S, Valinezhad A (2011) Genotypic characterization of Iranian camel (Camelus dromedarius) isolates of Echinoccocus granulosus. J Parasitol 97: 251-255. 